\title{
The proposed connection between clouds and cosmic rays: Cloud behaviour during the past 50-120 years
}

\author{
E. Pallé \& C.J. Butler \\ Armagh Observatory, College Hill, BT61 9DG. Armagh, N. Ireland; \\ epb@star.arm.ac.uk, cjb@star.arm.ac.uk
}

\begin{abstract}
Several authors have suggested that a link exists between the flux of galactic cosmic rays (GCR) and cloudiness. Here we review the evidence for such a connection from studies of cloud factors using both satellite and ground-based data. In particular, we search for evidence for the low cloud decrease predicted by the rising levels of solar activity and the low cloud-cosmic ray flux correlation indicated by satellite data. Sunshine and synoptic cloud records both indicate that the global total cloud cover has increased during the past century. This increase in total cloud cover argues against a dominating role by solar activity (via GCR) over cloud formation on centennial time scales. Either the predicted low cloud decrease has not occurred or the medium-high level cloud has increased to a greater extent than low cloud has decreased.
\end{abstract}

As there is no accurate long term data available on low cloud behaviour during the last century, we are not able to totally dismiss the link between GCR and cloudiness, but we list a number of arguments for and against the proposed cosmic ray-cloud connection.

Keywords: Clouds, Cosmic Rays, Climate, Solar Activity, Sunshine, Global Warming. 


\section{Introduction}

Many studies have shown correlations between solar activity and climate records, however, the physical mechanisms responsible remain obscure. One of the more straightforward mechanisms through which solar activity could influence climate is through changes in solar irradiance, but, whereas the solar constant is known to be variable, the amount of change detected in two decades of solar satellite observation is insufficient to account for more than a third of the global warming since the beginning of the century, and only a fifth over the most recent decades (Lean and Rind, 1998; Soon et al., 1996). The contribution of the Sun to global warming has therefore been frequently overlooked compared to that produced from the increased concentration of greenhouse gases in the atmosphere.

Several mechanisms have been proposed in the literature through which solar activity could alter climate including the influence of the extreme ultraviolet on the upper atmosphere leading to changes in the global circulation system (Haigh, 1994) and the influence of galactic cosmic rays (GCR) in the troposphere (Dickinson, 1975; Tinsley, 1989). In this review we are concerned with the suggestion that GCR may influence cloud formation processes.

Galactic cosmic rays are very energetic particles from outside the solar system which penetrate to the bottom of the troposphere, and are the dominant source of ionization above $1 \mathrm{~km}$ and below $60 \mathrm{~km}$ altitude. Below $1 \mathrm{~km}$, over land, radon is the primary ionising agent.

Both the flux and energy spectrum of GCR reaching the Earth's surroundings are modulated by the interplanetary magnetic field, which in turn is strongly influenced by the magnetic field of the Sun via the solar wind. Because GCR are ionized particles, they are deflected by the interplanetary magnetic field with the result that only some of the more energetic particles can penetrate to the inner solar system. When solar activity is at a maximum, the Sun's magnetic field is strong and fewer particles can 
reach the Earth; at solar minima the Sun's magnetic field is weaker and the particle flux grows. Thus modulation of the strength of the interplanetary magnetic field by solar activity gives rise to the well known inverse correlation between sunspot number (and other activity indicators) and the flux of GCR.

Dickinson (1975) suggested the possibility of a link between ionization produced by GCR and cloudiness. He stated that "solar-related fluctuations in some aspects of cloudiness could be important. Any such variations in cloudiness are likely to be related to variations in production of ionization near the tropopause by galactic cosmic rays,...". Moreover he added that "A 5-10\% change in thick cloud cover or a $0.5 \mathrm{~km}$ change in cloud heights with solar activity would probably have gone undiscovered up to now". Tinsley (1989) and Tinsley and Deen (1991) proposed a mechanism by which stratospheric ionization changes produced by cosmic rays would affect microphysical processes such as nucleation and growth of ice particles in high-level clouds, however this mechanism remains unproven. Following Dickinson and Tinsley's proposals, $\mathrm{Pu}-$ dovkin and Veretenenko (1995) found cloudiness decreases associated with Forbush decreases of galactic cosmic ray flux (sudden drops due to geomagnetic storms). The results indicated a reduction in the total cloud amount over the former USSR as observed from the ground in the 1-2 days period following strong Forbush decrease events. The results were found to be confined to a latitude belt of about 60-64 degrees North. More recently, Svensmark and Friis-Christensen (1997) suggested a possible relationship between the cloud cover over the oceans and the galactic cosmic ray flux. For their study, Svensmark and Friis-Christensen (1997) used the satellite cloud cover data from the ISCCP C2 dataset for the period 1983 to 1991. They found a correlation between the GCR and cloudiness over oceans excluding the tropics.

Clouds have two different effects on climate. They have a cooling effect due to an increase in the albedo and the reflection of short-wave solar radiation back to space and they trap the long-wave radiation emitted from the ground, thus also warming the climate. In general the clouds are believed to have a net cooling effect (Ramanathan et al., 1989), but the amount and sign of the cloud feedback will strongly depend on 
the cloud type and altitude.

Thus there are two possible mechanisms involving cloudiness change that could lead to a warming of the troposphere, (1) a reduction of low cooling clouds or (2) an increase in high warming clouds. If either or both of these possibilities resulted from the known increase in solar activity then a smaller residual warming would remain to be explained by the greenhouse effect.

Our aim in this paper is twofold: firstly, we review the credibility of the cosmic raycloud relationship and its predictions for climate, and secondly, we try to establish the cloud trends over the last 50 to 120 years from synoptic cloud data and cloud proxies to test the galactic cosmic ray-cloud connection (hereafter as GCR-CC) hypothesis. Information on past cloud changes is also valuable to test global circulation models, since the implications for the global climate can be of dramatic importance.

In the next section we examine the evidence for a GCR-CC as derived from satellite cloud data. In Section 3 we discuss the available ground-based synoptic observations and proxies for clouds. In section 4 we discuss the conclusions reached from examination of these meteorological records, and in section 5 we suggest some of the possible drivers of change. In section 6 the arguments for and against a GCR-CC, as well as the implication of such a connection for the climate change will be discussed.

\section{The GCR-CC from satellite cloud data}

The study of decadal to centennial climate variability requires long and reliable datasets. Unfortunately global cloud datasets are scarce and of relatively short duration. Satellite global coverage has only been available for the last 20-30 years, and has discontinuities due to the difficulty of combining data from satellites which have used different instrumentation.

In their initial study, Svensmark and Friis-Christensen (1997) used data from the 
ISCCP C2 (International Satellite Cloud Climatology Project) dataset from 1983-1991. They used satellite data only from geostationary satellites, discarding polar-orbiting satellite data in the belief that these were degraded by calibration problems. Cloud coverage over land areas was also discarded as the behaviour differed significantly from the clouds over oceans. They found a good correlation between the galactic cosmic ray flux and the total cloud cover over oceans which strengthened further when the tropical belt $\left(-22.5^{\circ}<\right.$ latitude $\left.<+22.5^{\circ}\right)$ was excluded. Because of the more effective shielding of galactic cosmic rays by the Earth's magnetic field at low latitudes, the improved correlation after removing the tropical areas was interpreted as additional evidence for a GCR-CC.

Svensmark and Friis-Christensen (1997) and Svensmark (1998) also used data from the Nimbus-7 matrix and the Defense Meteorological Satellite Program (DMSP). Plotted to scale with the ISCCP C2 data, these two datasets apparently confirmed the hypothesis of a cosmic ray-cloud connection. However the use of different inter-calibrated satellite datasets was strongly criticized by Kernthaler et al. (1999) and Kristjánsson and Kristiansen (2000), who showed that the ISCCP and DMSP datasets disagreed when overlapped.

On the other hand, the hypothesis of Svensmark and Friis-Christensen (1997) received some support from a paper by Menzel et al. (1996) which found a similarity in the variation of cirrus clouds and the GCR flux using data from the polar orbiting satellite HIRS (High-Resolution Infrared Radiometer Sounder). However later studies using this dataset suggest that the correlations of HIRS cloud products with cosmic rays and other solar activity parameters are not well understood (Hinton, 1999). Using 12-month running means (as the majority of authors in this subject have done) Hinton (1999), found a larger number of significant negative correlations between cloud and magnetic indices and sunspot number than positive correlations with cosmic rays, whereas the opposite is true for the ISCCP series. He also pointed out the difficulty of assessing the significance of the correlations for such short or filtered data series and the crucial role of the determination of the number of degrees of freedom. 
Serious doubts about the validity of the results by Svensmark and Friis-Christensen (1997) and apparent contradictions were also mentioned by Kernthaler et al. (1999) who tried to relate the different cloud types in the ISCCP C2 dataset with the galactic cosmic ray flux, particularly during the four years 1985-1988 during which the ISCCP data was believed to be well calibrated. They did not find any correspondence with a particular cloud type. They argued that, given that ionization produced by comic rays peaks at an altitude around 15 to $20 \mathrm{Km}$, high cirrus clouds should be the most affected. They included in their correlations the data from polar satellites which they believed were well calibrated with respect to the remaining satellites (Rossow et al., 1996). In the case of a GCR-CC, the inclusion of this data would be expected to improve the correlations; in fact the correlations worsened. Since the ionization produced by GCR is larger at high latitudes, this was taken as an argument against a possible cloud-cosmic ray relationship. The conclusions of Kernthaler et al. (1999) were in turn questioned by Svensmark and Friis-Christensen (1999) who pointed out that the former results are not reproduced with the later D2 data.

Kuang et al. (1998) also analysed the ISCCP C2 dataset, this time comparing the cloud amount with time against cloud optical thickness. They showed that these two parameters had a negative correlation, and argued that the combined effect of the two opposing variations may be a null effect on the cloud reflectivity.

Kristjánsson and Kristiansen (2000) compared the ISCCP C2 (1983-91) and D2 (198993) datasets, together with synoptic cloud cover over the oceans from Norris (1999, discussed in the next section) and the DMSP data, with the variation of the galactic cosmic ray flux. Their main finding was that the correlation coefficient between total cloud cover and cosmic ray flux diminished when data from the new ISCCP D dataset was included. They also rejected the suggestion that the correlation was maintained over any particular cloud type except possibly for the low marine clouds. The synoptic data indicated only a slight negative correlation with the cosmic ray flux. The DMSP data had a similar behaviour to the cosmic ray flux but, as the DMSP satellite detects only liquid cloud types over oceans, Kristjánsson and Kristiansen (2000) demonstrated 
that its data is not directly comparable with the ISCCP data for the time during which they overlap.

Contemporaneous with the Kristjánsson and Kristiansen (2000) paper, two further studies which looked for evidence of a GCR-CC were published by Pallé and Butler (2000) and Marsh and Svensmark (2000). They used the new re-calibrated ISCCP D2 dataset, which was believed to be superior and more reliable than the old $\mathrm{C}$ series and, more importantly, cover an additional three years. They also have a better agreement with ground-based cloud observations. From the cloud top temperature given by this dataset, Marsh and Svensmark (2000) found a correspondence between low cloud types and cosmic ray flux. No correlation was found for middle or high level clouds. The fact that the surface temperature and cloud top temperature also correlated with the GCR was taken as additional evidence for low cloud properties being affected by GCR. A correlation between low cloud factor and GCR was found independently by Pallé and Butler (2000). These authors also showed that the total cloud cover did not correlate with the cosmic ray flux after 1991/2. The analysis by Pallé and Butler (2000) covered different areas of the globe and found no strong latitudinal variation in the GCR$\mathrm{CC}$, except for the polar regions where the correlation dropped. Furthermore they argued, from the behaviour of clouds of liquid or ice droplets, that almost all variability in ISCCP D2 low cloud cover at low and middle altitudes can be accounted for by liquid phase clouds, and it is these alone which were responsible for the correlation. These results came from averaging over broad latitude bands, however, more recent results on the detailed geographical distribution of the correlations (Pallé and Butler, unpublished), using the ISCCP D1 data series, indicate that there exists a longitudinal as well as latitudinal dependence.

In brief the satellite data indicate the following:

-A strong correlation between low clouds as defined by the ISCCP D2 (1983-1994) dataset and cosmic ray fluxes $(99.8 \%$ significance level when using global averaged yearly means). A correlation between total cloud cover and cosmic rays occurs only 
during the restricted interval 1983-1991 (Pallé and Butler, 2000).

- There is a correspondence between water clouds measured by the DMSP and galactic cosmic ray flux. No other cloud type data is available from this source. (Svensmark and Friis-Christensen, 1997).

- There is a correspondence between cirrus clouds from the HIRS data (June 1989-May 1996) and cosmic ray flux (Menzel et al., 1996), however the significance level of this correlation has not been well established.

- Data from the Nimbus-7 cloud matrix for total cloud cover has been studied by Svensmark and Friis-Christensen (1997) with positive results for a cosmic ray-cloud relationship.

\subsection{The GCR-Cloud prediction}

If we suppose that the GCR-CC holds for low clouds over long periods of time then, due to an increase in solar activity and a more effective shielding of galactic cosmic rays in the heliosphere over the past 100 years (see Lockwood and Stamper, 1999), Pallé and Butler (2000) predicted a decrease in the low cloud cover during the past century. As there was no evidence to support a correlation with solar activity for other cloud types, it was assumed that they remained constant. Thus, if only low cloud varied with time due to a GCR-CC and all else were constant, even though total cloud cover does not correlate with GCR, a decrease in the total cloud since the late 19th century would be expected. The actual trend in low cloud factor for the last 100 years predicted by the correlation between GCR and low cloud is small $(\sim 1 \%)$, however, they estimated that it could have caused a positive climate forcing resulting in a rise in the global mean temperature of $0.27^{\circ} \mathrm{C}$ temperature increase. The cloud variability observed by satellite over one solar cycle is about $3-4 \%$.

The above analysis (Pallé and Butler, 2000), which was based on the observed correlation of low cloud factor with cosmic rays and solar activity indices has serious 
implications for our understanding of the causes of climate change as it could imply that most of the global warming during this period can be attributed to the combined direct (irradiance) and indirect (low cloud) effects of solar activity. However the predicted long-term trend in low cloud factor is small $(\sim 1 \%)$ and could easily be swamped by other factors affecting cloud cover not related to solar activity (e.g. a cloud redistribution towards more convective cloud types, or increase in cloud cover due to a rise in temperature, etc..). Reported trends in synoptic cloud observations and sunshine records are typically of the order of a few percentage points (1-3\%) change per decade, in this sense the cloud trends proposed by the GCR-CC are not unreasonable. A comprehensive forcing computation would require knowledge of the variability of clouds at all levels, and its geographical distribution. It is important, therefore, to examine any measured cloud factors over the past century.

\section{Long-term cloud data}

Since satellite-based cloud records do not extend for more than a couple of decades and calibration problems between existing datasets prevent a straight forward comparison, we have examined synoptic cloud observations and cloud proxy records from ground stations to study long-term cloud cover behaviour. The geographical and temporal coverage and brief details of any trends identified are given in Table 1.

So far, only negative results towards a GCR-CC have been reported when comparing ground based cloud observations and solar activity (Kristjánsson and Kristiansen, 2000; Wagner et al, 2001; Pallé and Butler, 2001). Only Udelhoffen and Cess (2001) found a correspondence between solar activity and total cloud cover over USA, but cloud changes were in phase with the sunspot number and not the GCR.

There is a fundamental difference between cloud cover as seen from the ground and observed by satellite. Ground observations are naturally weighted towards low clouds which are more easily observed from below, whereas satellite observations from above 
are weighted towards high cloud. Satellites cannot measure different cloud layers at the same time, so low clouds can be obscured by higher cloud in a similar way to the obscuration of high cloud when observed from the ground. For total cloud, the ISCCP cloud data agrees quite well with ground observations (Seze et al., 1986; Rossow, 1996, Pallé and Butler 2001), however, there are important differences when considering specific cloud types (Weare, 2000). For instance, the cloud classification scheme for the ISCCP data is not identical to that used by synoptic observers even though the same labels are used (Rossow, 1996). Thus discrepancies between satellite and ground based cloud observations are likely. Sunshine records are also of little help in this respect as they cannot distinguish between cloud types and total cloud is not correlated with GCR.

\subsection{Synoptic observations of cloud cover}

Synoptic observations of total cloud cover are made in many meteorological stations worldwide. The procedure is simple; at specified times of the day, the observer estimates the number of oktas (1/8 th of the sky) covered by clouds. A totally overcast sky is registered as 8 and a clear sky as 0 . At some stations, coverage by different cloud types is also recorded. It is evident that, to a greater or lesser extent, such estimates are observer dependent and therefore subject to systematic errors.

Observations of synoptic cloud cover over the oceans from volunteer ships since 1952 have been compiled and analysed by Norris (1999). Norris found that the global mean cloud cover over the oceans increased by $1.9 \%$ (sky cover) between 1952 and 1995 . Global mean low cloud cover was also observed to increase by $3.6 \%$ during the same period and trends in zonal mean cloud cover in $10^{\circ}$-lat bands between $60^{\circ} \mathrm{N}$ and $40^{\circ} \mathrm{S}$ were all found to be positive. Several possible artifacts were examined but it was considered unlikely that they could explain the observed inter-decadal variability. Norris concluded, however, that, in view of possible observer dependent differences, the trends cannot be accepted as definitive until they have been corroborated by related meteoro- 
logical parameters and satellite-based measurements.

Sun and Groisman (2000), have studied synoptic cloud cover variations over the former USSR from 1936 to 1990 . They find high cloud to be increasing and low cloud decreasing with the total cloud increasing over this period. Though for a continental, as opposed to a maritime climate region, these findings are pretty much in line with the trends suggested earlier by the the cosmic ray - low cloud correlation. On the other hand, though the total cloud trends are similar, it is difficult to reconcile the results by Norris (1999) and by Sun and Groisman (2000) on low clouds, other than to point out that

they refer to very different regions. It should be noted that the Sun and Groisman (2000) trends are very statistically significant whereas those by Norris (1999) are prone to many systematic effects such as might arise from a change in the latitude of preferred shipping lanes.

Similar reports to those of Norris (1999) and Sun and Groisman (2000), using cloud synoptic observations are listed in Table 1. McGuffie and Henderson-Sellers (1988) reported a synoptic cloud increase over Canada and the Canadian Arctic. HendersonSellers (1992) reported similar results over N. America, Australia, Europe and India. Only China (Kaiser, 1998) seems to differ from this pattern.

Whilst the behaviour of individual stations may differ strongly from the mean trends over a large area, it is notable that almost all studies of synoptic cloud cover show an increasing trend during the past century. No other general studies (involving more than one station) regarding synoptic clouds or cloud type variability are known to the authors.

\subsection{Sunshine data - a proxy for cloud cover}

One relevant proxy for cloud cover is sunshine duration. Daily records of the duration of bright sunshine have been obtained at four stations in Ireland since May 1880 using a standard Campbell-Stokes sunshine recorder (Observer's Handbook, 1982). Two are 
located in the east of Ireland; at Armagh Observatory and The Ordnance Survey Office, Phoenix Park, Dublin; one in the extreme west at Valentia Island/Cahirciveen, Co Kerry; and the fourth, in the midlands, at Birr Castle, Co Offaly. More information about the Irish sunshine data can be obtained at Pallé and Butler (2001).

The most prominent feature of the data, for all four sites, is a gradual decline in the total annual sunshine hours over much, if not all, of the 118 year period during which records have been obtained (see Figure 1). The effect is particularly conspicuous at the most westerly site of Valentia Island/Cahirciveen, on the County Kerry coast, where the number of sunshine hours has dropped by $\sim 20 \%$ since the end of the last century. If we plot the seasonal averages, the gradual decrease is seen in all stations in most seasons.

The sunshine data has been shown to be a very good proxy for total cloud cover over monthly to yearly time scales, at least over the Irish region (Pallé and Butler, 2001). Unfortunately the sunshine records are related only to the total cloud factor and do not give us any information on cloud type. It can also be argued that the sunshine records are a local measurement and that the observed trends are not necessarily similar for other areas. However, Pallé and Butler (2001) compared the variability of the ISCCP D2 satellite cloud records over Ireland and other areas of the globe and found that, over the period comprised by the data (July 1983-August 1994), cloudiness over Ireland behaves similarly, not only to the North Atlantic region as a whole, but to mid-latitude oceanic regions generally. Thus it seems that the sunshine factor over Ireland could be particularly useful in indicating wider trends.

A detailed comparison between the Irish sunshine records and the synoptic cloud cover over oceans near Ireland remains to be done. However, preliminary examination suggests an approximate agreement in percentage variability of sunshine and synoptic cloud cover over the last 50 years, of around 14\% (Pallé and Butler, 2001).

Though they cover a shorter time than the Irish records, similar results were reported by Stanhill (1998b) for sunshine records from Israel. Also, Angell (1990) and Angell 
et al. (1984) reported sunshine decreases and synoptic cloud increases during the period 1950-1988 for the United States. Changnon (1981) also reported sunshine decreases for mid-western United Sates during the more extended period 1901-1977. Both, Angell et al. (1984) and Changnon (1981), found the increasing trend in synoptic cloud to be larger than the decreasing trend in sunshine records, and this was attributed to an increase in the amount of thin cirrus which, though included in the synoptic cloud record, would be insufficiently opaque to reduce the instrumental sunshine duration.

\subsection{Radiation Decreases}

In Table 1 we refer also to the reduction in ground solar radiation levels reported at a world-wide distribution of sites during the last 40 years (Stanhill, 1998a and references

therein), suggesting a total cloud cover increase. However, we must be very careful when using such measurements to establish cloud trends, since radiation decreases have not been always accompanied by an increase in cloud cover and it is possible that changes in cloud type or atmospheric or cloud transparency could be responsible (Liepert, 1997).

\section{Discussion - the long-term cloud behaviour.}

If Irish and similar sunshine data are indeed relevant to the global trend they would indicate a rise in the total cloud factor since the late 19th century, in agreement with the synoptic cloud observations. The evidence for low cloud trends is less clear though a decrease in line with predictions from solar activity levels cannot be ruled out.

To estimate the possible contribution to global warming implied by the cosmic raycloud interaction, Svensmark (1998) predicted a total cloud cover decrease during the last century, which is not seen in the ground-based records. However it is now known from the new ISCCP D cloud products that only the low clouds vary in phase with the GCR. Pallé and Butler (2000) predicted a low cloud cover decrease since 1900 from the observed correlation of low clouds and cosmic rays in the interval 1983-1994 and known 
changes in solar parameters. If other cloud types remained constant this would imply a total cloud cover decrease over the last century. It is clear from Table 1 that, since total cloud has increased over the past century, either the decrease in low cloud cover has not occurred, or the supposition that the rest of the cloud types remain constant is not true.

A summary of the principal characteristics of two sets of satellite cloud data and two types of ground-based data (synoptic and sunshine) is given in Table 2. It is clear in the table how each series has its own characteristics. Parameters such as the time of measurement, the geographical coverage or even the problems the data present are not equivalent. This makes comparisons between the datasets difficult. Data obtained by one satellite, for example, does not always correspond to that from other satellites or from the ground, because they are not always looking at the same phenomena. In respect to the GCR-CC, we seem to be confronted by a contradiction. On one hand, satellite measurements indicate a correlation between low cloudiness and the GCR. This is based on global measurements for which the data is highly reliable. Unfortunately, these datasets are available for only a few years, and the significance of the correlations problematical and it is difficult to distinguish statistically between GRC and other solar-related parameters. Whereas ground-based observations have the requisite long duration, they do not correspond with the GCR and the trends argue against the GCRCC. In general ground-based data are less reliable than satellite observations since many factors (observer changes, station relocation,..) can introduce spurious trends in the series. In fact the only two 'clear' trends are: increasing liquid phase clouds over oceans from the DMSP (Defence Meteorological Satellite Project) satellites, and increasing cirrus clouds detected by the HIRS (High-resolution Infrared Radiation Sounder), and either or both of those trends may be a result of the short duration of the records.

The conclusions from Table 1 and 2 do not support the cosmic ray-cloud connection and its effects on the climate system. However, neither is there sufficient evidence of global low cloud trends over the past 100 years to refute it. All we can say is that, for the GCR-CC to be true, the amount of mid-level and high cloud would have to have 
increased in this period to a greater extent than low cloud decreased. At the present moment, there is no firm evidence for this, except possibly in the FUSSR (Sun and Groisman, 1999) and China (Kaiser, 1998).

The effect that an increasing trend in total cloud cover would have on the Earth's radiation balance is not clear. Overall clouds are believed to cool the climate and thus, to a first approximation, increasing cloudiness would result in a negative forcing to global temperatures. In other words, clouds could reduce global warming, acting as a thermostat. However, the net effect would depend on the altitude, the geographical distribution and physical properties of the cloud changes. Though, on average, thin high clouds warm and low clouds (or more specifically optically thick clouds) cool the climate, their combined effects are not necessarily additive; e.g. high thin clouds over low thick clouds will not warm. Even the temporal change in cloud occurrence can be important, as at night time all clouds have a warming effect. During the past century, much of the global warming has been attributed to milder temperatures at night (Houghton et al., 1995), as would be expected if night-time cloud cover alone had increased.

It is feasible, nonetheless, that if high clouds were to have increased due to some unknown cause over the past century to a greater extent than low cloud has decreased due to the operation of a GCR-CC, then the radiative forcing of the two changes would be compounded with the additional positive radiative forcing from solar irradiance changes, thereby all contributing to enhanced global warming. However, if the increase in total cloud reflected an increase in deep convective clouds, these would have an opposite forcing to any downward trend in low clouds and upward trend in solar irradiance. Until such time as reliable datasets can be assembled with information on the trends of different cloud types, these possibilities remain pure speculation and an encouragement to further research. 


\section{What is causing the total cloud increase?}

We have seen in the previous section how both synoptic cloud and sunshine data indicate that total cloud cover has been increasing over the last century. But what are the reasons for the change? Certainly the proposed connection between energetic particles entering the atmosphere and cloud formation would explain a decrease, but not an increase. Can the long term trend in total cloud result from a mean air and sea surface temperature increase? Little is known about the effects that a change in temperature will have on clouds. Global circulation models predict a cloud factor decrease when climate warms (Cess et al., 1996) however, even though the models predict an uneven reduction in cloudiness and clouds are still poorly parameterised, such a reduction has not been reported so far. On the other hand, following the rise in sea-surface temperatures which have accompanied global warming (Reid, 1987), an increase in cloud formation might follow from an increase in evaporation rates. A progressive moistening of the atmosphere has been reported by Wentz and Schabel (2000), however, warmer air is able to hold more water vapour before saturation and thus relative humidity could have remained constant. As cloud formation depends critically on relative humidity, an increase in the water vapour content of the atmosphere (absolute humidity) would not necessarily lead to an increase in cloud factor.

Cloud amounts over Canada have been reported to increase over practically all the regions where temperatures have risen (McGuffie and Henderson-Sellers, 1988). However station records from the Canadian Arctic showed cloud changes which seem decoupled from temperature changes. For the United States, Angell et al. (1984) found an appreciable tendency for cloudiness to be above average and sunshine to be below average in years of warm sea surface temperature in the equatorial eastern pacific (El Niño). Henderson-Sellers (1992) also noticed a tendency for total cloud amount to be greater in warmer conditions.

An increase in tropospheric aerosols could also give rise to increased cloud formation. However (Norris, 1999) stated that the $10^{\circ}$-lat band trends in synoptic cloud observa- 
tions between $60^{\circ} \mathrm{N}$ and $40^{\circ} \mathrm{S}$, are generally larger for the Southern Hemisphere and Tropics than for the mid-latitude Northern Hemisphere. For Norris (1999), this argued against attribution of increased cloud cover to increased anthropogenic aerosols, and suggested that it is possible that global cloud cover is responding to some other global parameter, perhaps temperature.

Pallé and Butler (2001) found a correlation between the sunshine records over Ireland and the solar cycle length. The solar cycle length is defined as the time from minima to minima (or maxima to maxima) of the sunspot number, it is related to solar activity in the sense that generally shorter solar cycles are accompanied by higher levels of activity (higher sunspot maxima). Since the sunspot cycle length was shown to vary in phase with the NH air temperature (Friis-Christensen and Lassen, 1991), and that relationship was confirmed for an Irish (Armagh) temperature dataset by Butler and Johnson (1996), they concluded that in the vicinity of Ireland at least, it seems likely that decreased sunshine hours (increased cloudiness) result from the increased temperatures associated with global warming, rather than to any kind of direct solar influence on clouds.

Another effect to take into consideration is the effect of increased aircraft traffic. Changnon (1981) indicated that localized shifts to more cloudiness in zones of increased air traffic since 1960, suggest anomalous changes related to jet-induced cirrus. However, Menzel (1996) found that the increasing trend in cirrus cloud cover detected by the HIRS was no more noticeable over areas with heavy air traffic than over areas with low air traffic.

In many of the sites where a decrease in total solar irradiance has been found, an increasing trend of cirrus cloud formation has also been detected (Liepert, 1997). A shift from stratiform to higher frequencies of convective clouds has also been observed (Liepert, 1997; Stanhill, 1998a). Finally, another possible explanation for the long-term cloud change could be through shifts in weather patterns such as the location of storm tracks.

Even though none of the sunshine or synoptic cloud records are exempt from the 
possibility of systematic errors, the agreement between the different and diverse data series in their apparent trends reinforces our opinion that the trend towards increasing cloudiness is real and, probably, global.

\section{Does a cosmic ray - cloud cover connection exist?}

\subsection{Arguments in favour}

In light of the arguments explored in previous sections it seems clear that a direct relationship between total cloud cover and cosmic ray flux is unlikely. The most reliable satellite dataset, the ISCCP D2 (Pallé and Butler, 2000), the synoptic cloud cover (Norris, 1999; Kristjánsson and Kristiansen, 2000) and the sunshine records (Pallé and Butler, 2001; Wagner, 2001) support that conclusion. For different cloud types the situation is different. Clearly the favourite candidate for a GCR-CC is the low cloud cover. Even though discarded at first by Kernthaler et al. (1999) in the ISCCP C2 dataset, the correlation is clear in the new ISCCP D2 dataset (Pallé and Butler, 2000; Marsh and Svensmark, 2000).

The correspondence between the DMSP cloud records and the galactic cosmic ray flux roughly agrees with the findings of the ISCCP D2 data. The DMSP satellite measures only liquid clouds over the oceans, which consist mainly of low-level clouds. However during the period of overlap the two series do not totally correspond in all respects (Kristjánsson and Kristiansen, 2000). The correspondence between cloud top temperature and surface temperature with the GCR (Marsh and Svensmark, 2000) also supports the GCR-CC.

The Earthshine project which monitors the Earth's albedo and is currently underway at Big Bear Solar Observatory may impact on our discussion. A global and absolutely calibrated albedo for the Earth can be determined by measuring the amount of sunlight reflected from the Earth to the Moon and back. Preliminary results seem to support 
a lower planetary albedo at solar activity maximum (Goode et al., 2001) which would support the GCR-CC, however it is too early and the time span too short to reach any definitive conclusion.

\subsection{Arguments against}

The main argument against the GCR-CC, is the lack of an established physical mechanism. Turco et al. (1998) and Yu an Turco (2001) have proposed a mechanism "ion-ion recombination", by which ionization produced by GCR could facilitate aerosol production and growth in the lower troposphere. Svensmark and Friis-Christensen (1997) and Marsh and Svensmark (2000) have speculated that it is not unreasonable to imagine that systematic variations in cosmic ray ionization could influence cloud properties via this mechanism.

An alternative mechanism to explain a cosmic ray influence in ice clouds has been proposed by Tinsley et al. (2000) and references therein. However his mechanism refers only to ice clouds (mainly cirrus), which are not seen to vary with the cosmic ray flux, except for the HIRS data which covers only a very short time. It is difficult also to explain a cloud increase during the past century using this mechanism.

Concern arises also from the temperature records. If an indirect solar forcing coming from the GCR-CC acts on the climate system, a stronger variability of the mean temperature over the $\sim 11$ year cycle would be expected. Though ocean thermal inertia damping is likely to be important here, a small amplitude signal would still be expected.

In addition, there is the unexplained latitudinal distribution of the low cloud-cosmic ray correlation. On account of the latitude-differential shielding of the Earth's magnetic field, the correlation would be expected to increase poleward, whereas in fact it remains almost constant, except for the poles where it has a small drop (Pallé and Butler, 2000). Pallé and Butler (2000) suggested that the observed latitude dependence could result

if only liquid phase clouds were amenable to the influence of cosmic rays. Ice-phase 
clouds, more abundant at the poles or high altitudes, would be unaffected by cosmic rays, thereby leading to a lack of correlation with clouds at both high altitudes and high latitudes. However in a recent study, a longitudinal as well as latitudinal dependence of the correlation has been found by Pallé and Butler (unpublished) which no longer supports the suggestion that only liquid clouds phase clouds are affected by GCR.

Finally, we note possible changes in cloud reflectivity and opacity. Kuang et al. (1998) analysed the cloud optical thickness and reflectivity changes for the period 1983-1991 and found that, even though the cloud amount changed with the cosmic ray flux, the cloud optical properties changed too, compensating for the changes in cloud amount. As a consequence, even though the cloud factor may change, the effect on the radiation budget could be negligible.

A further complication in any cloud influence on climate was recently raised by Del Genio and Wolf (2000). Based on three years of low cloud data, Del Genio and Wolf (2000) found that low clouds thin when temperatures rise. As a result, if the temperature increases over the next decades, low clouds could become thinner, reduce their albedo and thus contribute a positive feedback to global warming. If this were true, part of the global warming experienced during the last century might have come from a thinning of low cloud, further reducing the contribution required from the greenhouse effect.

\section{Conclusions}

There appears to be an statistically significant (99.8\% significance level) correspondence between the low cloud cover as seen from modern satellite data and the galactic cosmic ray flux. However the duration of the dataset is short and leaves margin for many uncertainties when trying to establish long-term behaviour of the cloud cover.

In order to assess the validity of prediction over long time scales, some synoptic cloud observations and cloud proxies have been compared. The sunshine records and the 
synoptic cloud cover over many areas of the Earth agree that the total cloud cover has been increasing over the last century. This trend goes in the opposite direction to the trend predicted for low clouds by the cosmic ray-low cloud correlation. Though the accuracy of some of the datasets is open to question, the agreement in the observed trends suggests that they are real.

The GCR-CC cannot be positively discounted at this time, at least for the last 20 years, for which satellite data is available. The reason is that no accurate data for low cloud cover is available to ascertain whether or not the correlation is maintained further back in time (see Table 2). However, two facts: (1) the lack of correlation between total cloud cover and GCR and (2) the general increase in total cloud cover over the last century showed by several studies of cloud and cloud-proxy data, indicate that it is likely that some mechanism(s) other than GCR are the main drivers of cloud cover changes on century time scales. Some possible mechanisms for the cloud increase are discussed; the most promising of which seems to be a rise in evaporation rates following the rise in sea-surface temperatures. However, this will not necessarily cause an increase in cloudiness if air temperatures warm sufficiently to prevent a rise in relative humidity.

It seems likely therefore, that the simplified scenario of cloud amount governed by solar activity (GCR) alone, is not valid as trends in cloudiness go the opposite way to predicted. If real, the radiative effects of clouds affected by the GCR-CC, will have to be combined with the radiative effects of clouds influenced by other mechanism(s). A net increase in total cloud cover (not taking into account a shift in cloud types) during the last 100 years would lead, to a first approximation, to a negative radiative forcing. With a net radiative forcing by clouds between 3 and 5 times larger than that from a doubling of $\mathrm{CO}_{2}$ concentration in the atmosphere (Henderson-Sellers, 1992), the importance of cloud factor changes will depend on the variability of the different cloud types and their geographical distribution. It is also clear that cloud factors have been changing during the recent past, and until the cloud behaviour on temporal and geographical scales is understood (via long and reliable datasets) and assimilated into global circulation models, predictions will be seriously handicapped. 


\section{Acknowledgements}

We would like to thank Dr. J. Haigh and an anonymous referee for their comments on an earlier version of this manuscript. The sunshine data for Dublin, Birr and Valentia Island/Cahirciveen were kindly provided by D. Fitzgerald of Met Eireann, Dublin. Research at Armagh Observatory is grant-aided by the Department of Culture, Arts and Leisure for Northern Ireland.

\section{References}

Angell, J.K., 1990. Variation in United States cloudiness and sunshine duration between 1950 and the drought year of 1988. J. Clim. 3, 256-308.

Angell, J.K., Korshover, J., Cotton, G.F., 1984. Variation in United States cloudiness and sunshine, 1950-82. J. Clim. \& App. Met. 23, 752-761.

Butler, C.J., Johnson, D.J., 1996. A provisional long mean air temperature series for Armagh Observatory. JASTP 58, 1657-1672.

Cess, R.D., et al.(34), 1996. Cloud feedback in atmospheric circulation models: an update. J. Geophys. Res. 101, 12791-12794.

Changnon, S.A., 1981. Midwestern cloud, sunshine and temperature trends since 1901: possible evidences of jet contrail effects. J. App. Met. 20, 496-508.

Del Genio, A.D., Wolf, A.B., 2000. The temperature dependence of the liquid water path of low clouds in the southern Great Plains. J. Clim. 13, No. 9, 3465-3486.

Dickinson, R.E., 1975. Solar variability and the lower atmosphere. Bulletin American Meteorological Society 56, 1240-1248.

Friis-Christensen, E., Lassen, K., 1991. Length of the solar cycle, an indicator of solar activity closely associated with climate. Science 254, 698-700.

Goode, P.R., Qiu, J., Yurchyshyn, V., Hickey, J., Chu, M-C., Kolbe, E., Brown, C.T., 
Koonin, S.E., 2001. Earthshine observations of the Earth's reflectance. Geophys. Res. Letts. 28, 1671-1674.

Haigh, J.D, 1994. The role of stratospheric ozone in modulating the solar radiative forcing. Nature 370, 544-546.

Henderson-Sellers, A., 1992. Continental cloudiness changes this century. GeoJournal $\mathbf{2 7 : 3 ,} 255-262$.

Hinton, B.B., 1999. Solar Cycle Cloud Variations. Space Science and Engineering Center Internal Report. University of Wisconsin-Madison, 9 Mach 1999.

Houghton, J.T., Meira Filho, L.G., Callander, B.A., Harris, N., Kattenberg, A., Maskell, K., (Eds). Climate Change 1995. The science of climate change, 1995. Cambridge University Press, p 437.

Kaiser, D.P, 1998. Analysis of total cloud amount over China, 1951-1994. Geophys. Res. Letts. 25, 3599-3602.

Kernthaler, S.C., Toumi, R., Haigh, J.D., 1999. Some doubts concerning a link between cosmic ray fluxes and global cloudiness. Geophys. Res. Letts. 26, 863-865.

Kuang, Z., Jiang, Y., Yung, Y.L., 1998. Cloud optical thickness variations during 1983-1991: solar cycle or ENSO? Geophys. Res. Letts. 25, 1415-1417.

Kristjánsson, J.E., Kristiansen, J., 2000. Is there a cosmic ray signal in recent variations in global cloudiness and cloud radiative forcing? J. Geophys. Res. 105, 1185111863.

Liepert, B.G., 1997. Recent changes in solar radiation under cloudy conditions in Germany. Int. J. Clim. 17, 1581-1593.

Lean, J., Rind, D., 1998. Climate forcing by changing solar radiation. J. Clim. 11, 3069-3094.

Lockwood, M., Stamper, R., 1999. Long-term drift of the coronal source magnetic flux 
and the total solar irradiance. Geophys. Res. Letts. 26 (16), 2461-2464.

Marsh, N.D., Svensmark, H., 2000. Cosmic rays, clouds and climate. Space Science Review (in press)

McGuffie, K., Henderson-Sellers, A., 1988. Is Canadian cloudiness increasing? AtmosphereOcean 26, 608-633.

Menzel, W.P., Wylie, D.P., Strabala, K.I., 1996. Seven years of global cirrus cloud statistics using HIRS. IRS'96: Current problems in atmospheric radiation, Smith and Stammes (Eds), ISBN 0-937194-39-5.

Norris, J.R., 1999. On trends and possible artifacts in global ocean cloud cover between 1952 and 1995. J. Clim. 12, 1864-1870.

Observers Handbook, 4th Edition, 1982. UK Meteorological Office (Eds). Her Majesty's Stationery Office, London, p 153.

Pallé, E., Butler, C.J., 2000. The influence of cosmic rays on terrestrial clouds and global warming. Astron. 6 Geophys. 41, 18-22.

Pallé, E., Butler, C.J., 2001. Sunshine records from Ireland, cloud factors and possible links to solar activity and cosmic rays. Int. J. Clim. 21, 709-729.

Pudovkin, M.I., Veretenenko, S.V., 1995. Cloudiness decreases associated with Forbushdecreases of galactic cosmic rays. J. Geophys. Res. 57, 1349-1355.

Ramanathan, V., Cess, R.D., Harrison, E.F., Minnis, P., Barkstrom, B.R., Ahmad, E., Hartmann, D., 1989. Cloud-radiative forcing and climate. Results from the Earth Radiation Budget Experiment. Science 243, 52.

Reid, G.C., 1987. Influence of solar variability on global sea surface temperatures. Nature 129: 142-143.

Rossow, W.B., Walker, A.W., Beuschel, D.E., Roiter, M.D., 1996. 1996: International Satellite Cloud Climatology Project (ISCCP) Documentation of New Cloud Datasets. 
WMO/TD-No. 737, World Meteorological Organization.

Seze, G., Drake, F., Desbois, M., Henderson-Sellers, A., 1986. Total and low cloud amounts over France and southern Britain in the summer of 1983: comparison of surface-observed and satellite retrieved values. Int. J. Rem. Sens. 7, 1031-1050.

Stahle, D.W., Cleaveland, M.K., Cerveny, R.S., 1991. Tree-ring reconstructed sunshine duration over central USA. Int. J. Clim. 11, 285-295.

Stanhill, G., 1998a. Estimation of direct solar beam irradiance from measurements of the duration of bright sunshine. Int. J. Clim. 18, 347-354.

Stanhill, G., 1998b. Long-term trends in, and spatial variations of, solar irradiance in Ireland. Int. J. Clim. 18, 1015-1030.

Sun, B., Groisman, P.Y., 2000. Cloudiness variations over the Former Soviet Union. Int. J. Clim. 20, 1097-1111.

Soon, W.H., Posmentier, E.S., Baliunas, S.L., 1996. Inference of solar irradiance variability from terrestrial temperature changes, 1880-1993: An astrophysical application of the Sun-Climate connection. Astrophys. J. 472, 891-902.

Svensmark, H., Friis-Christensen, E., 1999. Reply to comments on "Variation of cosmic ray flux and global cloud coverage - a missing link in solar-terrestrial relationships". $J$. Atmos. Solar-Terrest. Phys. 62, 79-80.

Svensmark, H., Friis-Christensen, E., 1997. Variations of Cosmic Ray Flux and global cloud coverage. A missing link in solar-climate relationships. J. Atmos. Solar-Terrest. Phys. 59, 1225-1232.

Svensmark, H., 1998. Influence of cosmic rays on Earth's climate. Phys. Rev. Letts. $\mathbf{8 1}, 5027-5030$.

Tinsley, B.A., Rohrbaugh, R.P., Hei, M., Beard, K.V., 2000. Effects of Image Charges on the Scavenging of Aerosol Particles by Cloud Droplets, and on Droplet Charging 
and Possible Ice Nucleation Processes. J. Atmos. Sci. 57, 2118-2134.

Tinsley, B.A., Brown, G.M., Scherrer, P.H., 1989. Solar variability influences on weather and climate - possible connections through cosmic-ray fluxes and storm intensification. J. Atmos. Solar-Terrest. Phys. 94, 14783-14792.

Turco, R.P., Zhao, J., Yu, F., 1998. A new source of tropospheric aerosols: Ion-ion recombination. Geophys. Res. Letts. 25, 635-638.

Udelhofen, P.M., Cess, R.D., 2001. Cloud cover variations over the United States: An influence of cosmic rays or solar activity? Geophys. Res. Letts. 28, 2617.

Wagner, G., Livingstone, D.M., Masarik, J., Muscheler, R., Bee, J., 2001. Some results relevant to the discussion of a possible link between cosmic rays and the Earth's climate. J. Geoph. Res. 106(D4), 3381-3387.

Weare, B.C., 2000. Near-Global observations of low clouds.J. Clim. 13, 1255-1268.

Wentz, F.J., Schabel, M., 2000. Precise climate monitoring using complementary satellite data sets. Nature 403, 414-416.

Yu, F.Q., Turco, R.P., 2001. From molecular clusters to nanoparticles: Role of ambient ionization in tropospheric aerosol formation. J. Geoph. Res. 106(D5), 4797-4814. 
Figure 1: Mean annual sunshine factor for the four Irish sites (18811998). 


\begin{tabular}{lcccc}
\hline Dataset & Total Cloud & Low Cloud & High Cloud & Period \\
\hline & & & & \\
Ground Data Trends & & & & \\
\hline${ }^{1}$ Sunshine in Ireland (decrease) & Increase & - & - & $1881-1998$ \\
${ }^{2}$ Sunshine in Israel (decrease) & Increase & - & - & $1979-1995$ \\
3Sunshine over midwestern USA (decrease) & Increase & - & - & $1901-1977$ \\
${ }^{4}$ Sunshine over USA (decrease) & Increase & - & - & $1950-1988$ \\
${ }^{5}$ Sunshine Central USA (tree-rings) & Stable & - & - & $1700-1980$ \\
${ }^{6}$ Synoptic Clouds over FUSSR & Increase & Decrease & Increase & $1936-1990$ \\
${ }^{7}$ Synoptic Cloud over Oceans & Increase & Increase & - & $1952-1995$ \\
${ }^{8}$ Synoptic Cloud over Australia & Increase & - & - & $1900-1990$ \\
${ }^{9}$ Synoptic Cloud over North America & Increase & - & - & $1900-1990$ \\
${ }^{10}$ Synoptic Cloud over India & Increase & - & - & $1900-1990$ \\
${ }^{11}$ Synoptic Cloud over Europe & Increase & - & - & $1900-1990$ \\
${ }^{12}$ Synoptic Cloud over Canada & Increase & - & - & $1900-1954$ \\
${ }^{13}$ Synoptic Cloud over Arctic Canada & Increase & - & - & $1900-1980$ \\
${ }^{14}$ Synoptic Cloud over China & Decrease & - & - & $1954-1994$ \\
${ }^{15}$ Ground-based solar radiation (various) & Increase & - & - & $1960-2000$ \\
\hline & & & & \\
Satellite Data Trends & & - & Increase & $1985-1988$ \\
${ }^{16}$ ISCCP C2 & Stable & Stable & Stable & $1983-1994$ \\
${ }^{17}$ ISCCP D2 & Increase & - & - & $1988-1998$ \\
${ }^{18}$ DMSP (water clouds over Oceans) & - & - & Increase & $1989-1996$ \\
${ }^{19}$ HIRS (only Cirrus) & &
\end{tabular}

Table 1: Sunshine records and synoptic cloud data compilations from the ground. Satellite cloud measurements from different satellites are also displayed, however the short duration of those records makes them unsuitable for long-term studies. Cloud types reported as stable, as in the case of ISCCP C2 and D2, are in fact quite variable over the duration of the data, and a definite trend cannot be clearly established. References: ${ }^{1}$ Pallé and Butler (2001); ${ }^{2}$ Stanhill (1998a); ${ }^{3}$ Changnon (1981); ${ }^{4}$ Angell (1990); ${ }^{5}$ Stahle et al. (1991); ${ }^{6}$ Sun and Groisman (2000); ${ }^{7}$ Norris (1999); ${ }^{8}$ Henderson-Sellers (1992); ${ }^{9}$ Henderson-Sellers (1992); ${ }^{10}$ Henderson-Sellers (1992); ${ }^{11}$ Henderson-Sellers (1992); ${ }^{12}$ McGuffie and Henderson-Sellers (1988); ${ }^{13}$ McGuffie and Henderson-Sellers (1988); ${ }^{14}$ Kaiser (1998); ${ }^{15}$ Stanhill, 1998 b and therein; Liepert, 1997; ${ }^{16}$ Kernthaler et al., 1999; ${ }^{17}$ Pallé and Butler, 2000; ${ }^{18}$ Kristjánsson and Kristiansen, 2000; ${ }^{19}$ Menzel et al., 1996. 


\begin{tabular}{|c|c|c|c|c|}
\hline Question & ISCCP-D2 & DMSP & Synoptic Clouds & Sunshine \\
\hline Data reliability & Good & Good & Not Instrumental & Good \\
\hline Time of observations & Day+Night & Day only & Day only & Day only \\
\hline Geographical Coverage & Global & Oceans & Local & Local \\
\hline Main Problem & Duration & Duration & Spurious trends? & - \\
\hline Is there a trend in cloud cover? & - & - & Increase & Increase \\
\hline Can we distinguish cloud types? & Yes & Water clouds & Total/Low & No \\
\hline Is total cloud correlated with GCR? & No & - & No & No \\
\hline Is high cloud correlated with GCR? & No & - & - & - \\
\hline Is mid cloud correlated with GCR? & No & - & - & - \\
\hline Is low cloud correlated with GCR? & Yes & Yes? & No & - \\
\hline
\end{tabular}

Table 2: A brief summary of the distinguishing features of two sets of satellite cloud data and the two types of ground-based cloud data. See text for details. 

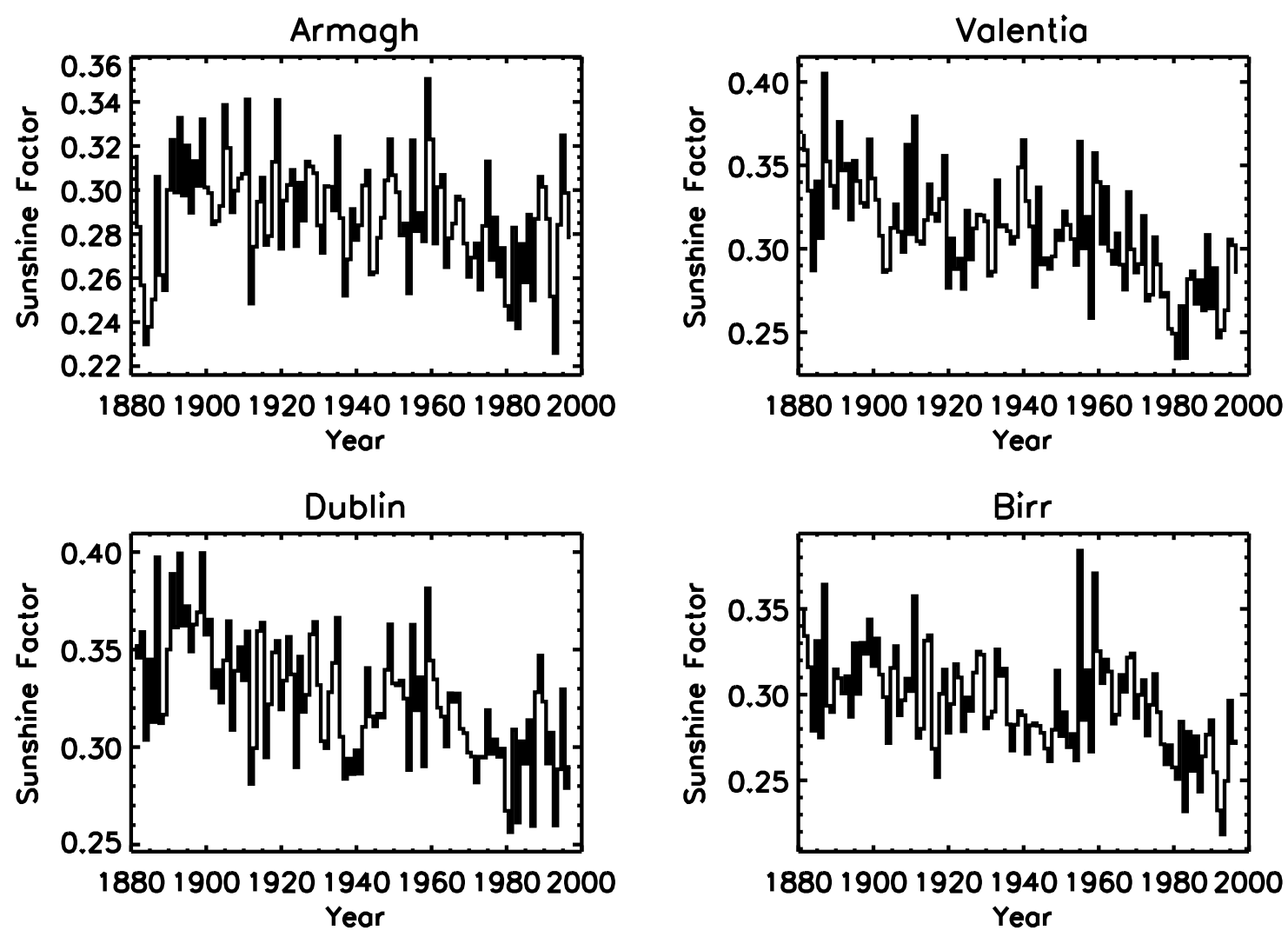

Figure 1: 